\title{
The role of magnetic component of a strong light field in electrostrictive effect
}

\author{
L.V. Poperenko, V.V. Prorok, S.G. Rozouvan, I.A. Shaykevich \\ Taras Shevchenko National University of Kyiv, 64/13, Volodymyrska st. 01601, Kyiv, Ukraine \\ E-mail: sgr@univ.kiev.ua
}

\begin{abstract}
Electrostriction forces during laser ablation have been studied both theoretically and experimentally. The components of electroctrostriction force for inhomogeneous electromagnetic field near a substrate were proposed to be taken similarly to those in gases within nonresonant spectral region. Nonzero Lorentz force in standing light wave was found to be responsible for different morphology of the nanostructured surface as compared to etching the transient substrate. Our experiments were performed using the femtosecond laser focused on polished glass and Al-coated glass surfaces. The treated surfaces were studied using atomic force microscopy with the spatial resolution of $30 \mathrm{~nm}$. Nanoscale patterning of the etched surface spots was explained in the frames of theoretical modeling. Possible spatial locations of electrostriction force components in the Gauss profile laser beam have been also discussed.
\end{abstract}

Keywords: electrostriction pressure, Lorentz force, femtosecond laser pulses, nanoparticles.

doi: https://doi.org/10.15407/spqeo21.02.160

PACS 42.50.Ct, 77.65.-j, 79.20.Eb, 81.15.Fg

Manuscript received 29.05.18; revised version received 05.06.18; accepted for publication 27.06.18; published online 03.07.18.

\section{Introduction}

Laser ablation has become a known technique for nanostructure fabrication after introduction of Ti:sapphire femtosecond laser systems [1]. Femtosecond laser light interacts with a target during $10^{-13} \ldots 10^{-14} \mathrm{~s}$ intervals. During the interval, the intensity of electromagnetic field is increased drastically in a tiny volume that consecutively results in temperature and electrostrictive pressure rising. The mixture of light induced ultrafast processes can produce a difference in nanostructures on the target surface or in the target bulk. Ultrafast pulsed laser deposition on $\mathrm{ZnO}$ target created variety of nanoforms including nanoparticles and nanorods [2]. Multi-pulse ablation of bulk gold sample results in production of nanostructures caused by combinations of nano-, micro-, and macro-structural surface modifications [3]. Femtosecond laser pulses train that was applied for ablation enables a broad and continuous tunability over the material morphologies ranging from nanoparticle aggregates to epitaxial thin films [4]. Laser ablation in liquid produces nanoparticles with variety of shapes from spherical domination to the tetragonal ones [5]. Ablation of solid surfaces fabricates nanoparticle aerosols clouds with particles in the few nanometer size range [6]. Surface etching by a nanosecond laser results in specific properties of nanoparticle film such as low melting temperature and reduced heat losses [7]. Pulsed-laser ablation of active glass was able to produce nanoparticles exhibiting such properties as upconversion pumped by near-infrared wavelengths and emitting visible light [8]. By contrast to projects dealing with wide variety of produced nanostructural forms, research groups produce more narrow aimed studies. A simple model theoretically describes the processes involved in irradiation of solid targets by using femtosecond laser pulses and predicts the optimal target and laser parameters for nanoparticles synthesis, which was developed and experimentally checked [9]. Consecutive treatment after laser irradiation by Ti:sapphire laser delivering $80 \mathrm{fs}$ pulses allowed predictably changing the properties of $\mathrm{TiO}_{2}$ 
nanostructures grown on Si [100] substrates [10]. The nanoparticles obtained as a result of laser ablation process showed more complicated structural properties after consecutive treatment in Se-evaporated atmosphere [11]. Nanostructural properties of the obtained nanoparticles have been studied by variety of experimental techniques including electron microscopy, atomic force microscopy, etc. The obtained high resolution images [5] describe nanoparticles both having no ideal spherical shape and non-uniform in size [12].

The processes resulted in nanostructural patterning of the irradiated surface are not completely explained. For example, focused femtosecond pulses should work as "optical tweezers" [13] keeping the nanoparticles in the focal point preventing them from removing out there. However, numerous experiments register large areas covered by nanoparticles on the samples surfaces aside from ablated tracks. The basic description of the interaction femtosecond laser pulse with a solid substrate includes the electrostriction mechanism that was found to be responsible for formation of laser-induced periodic surface structures [14]. On the other hand, the classical electrostriction relationship was found to be insufficient for such structure formation description and the authors introduced Maxwell stress tensor [15] for correct explanation of their results.

The variety of the works aimed at studying laser pulse interaction with a substrate at the nanoscale level with clear practical output requires some unification of the basic approaches for laser etching description. The goal of this research is to study features of laser etching mechanisms from the viewpoint of basic electromagnetic theory.

\section{Theoretical}

As it was found earlier [16], electrostriction force induced by electromagnetic wave has different properties as compared to constant electric field case. The latter case results in the well known ratio where electrostriction pressure depends on the density of electric field energy. If electric field produces dipoles in gas or liquid, it leads to changing the shape and position of the dielectric as a result of electrostriction pressure. The total process is considered to be static (otherwise, we have a system with a nonzero magnetic field). In the case of electromagnetic wave that propagates through a dielectric, the electromagnetic field can result in dipoles motion. For example, an inhomogeneous electric field that interacts with a dipole in gases can produce an additional to the "classic" case nonzero Coulomb force [16]. The dipole motion creates additional pressure, and the final ratio for electrostriction pressure in isotropic media is not a scalar value any more.

Let us consider an isotropic media with zero magnetic succeptibility in a monochromatic electromagnetic field. For a steady case of the monochromatic field, both magnetic and electric vectors can be presented as $E=E_{0}(\vec{r}) \sin (\omega t)$ and
$H=H_{0}(\vec{r}) \sin (\omega t)$, respectively. The classical electric dipole with the polarizability $\alpha$ having two charges separated by a distance $l$ oscillates with the frequency $\omega$ : $l(t)=l_{0} \sin (\omega t)=\alpha E_{0} /(q \sin \omega t)$.

Electrostriction forces in isotropic media are proportional to $E_{0}^{2}$. In our early work [16], we show that dipole irradiated by spatialy inhomogenous monohromatic light wave experiences an additional force that can be described as:

$\vec{F}_{E S}=\vec{E} \operatorname{div} \vec{D}$

Eq. (1) was derived describing the kinetics of a single dipole, though it can be understood from a general reasons considering a bulk media. If we take a charge density in dielectric media at a point, it is equal to $\operatorname{div} \vec{D}$. Finding Coulomb force on the charge as the product of the charge and the electric field, we obtain Coulomb force per volume ratio similar to Eq. (1). The additional force of Eq. (1) acts during a short time of laser pulse duration causing, for example, polarizationdependent electrostriction induced gratings in isotropic media [17]. However, historically electrostriction term was introduced for dielectric media in constant electrostatic field. The basic ratio for such electrostriction pressure was derived from the energy conservation law for steady state systems. In our case it is related with fast oscillations of electric and magnetic components of propagating light, and we obtain an additional pressure due to the field spatial inhomogeneity. We are continuing to name such pressure as electrostriction, like to that used in literature, though technically it is caused by electromagnetic wave and should be described by respective formulas.

One should carefully apply Eq. (1) for ultrafast processes of dipole oscillation. For example, if the laser frequency fits the ground-excited state energy gap for dielectric, we obtain more steady state electrostriction pressure because excited molecular levels are populated and cannot oscillate with the light frequency. Another problem may be a short laser pulse duration. For example, for focused femtosecond laser pulses its duration is order of magnitudes less then characteristic times for hydrodynamic equations solutions. For nanosecond pulses, the processes of thermal and mass diffusion match focal point dimension, if taking into account the speed of sound, flow velocities, etc. [17]. A simple criteria, if the system reaches steady state regime, is follows: the focal point volume dimensions should be less than the product of laser pulse duration and the speed of sound in this media. According to Eq. (1), laser pulse produces gradient of electromagnetic fields, which result in dipoles flow. After a few tens nanosecond time, the steady state regime is established, and the pressure in the media satisfies the Bernoulli equation. For example, if moving dipoles localized in the focal point cause dynamic pressure, it results in lowering the static pressure in this point. 
The total force applied to the dipole by electromagnetic field is a sum of Coulomb and Lorentz forces. In the latter case, we take into account only the electron speed, neglecting the Lorentz force that acts on positive ion because of its relatively low velocity (due to drastical difference between electron and ion masses).

$$
\begin{aligned}
& \vec{F}_{\text {Lorentz }}=q[\vec{V}(\vec{r}) \times \vec{B}(\vec{r})] \sin \omega t \cos \omega t= \\
& =\omega \alpha[\vec{E}(\vec{r}) \times \vec{B}(\vec{r})] \sin \omega t \cos \omega t, \\
& \left\langle\vec{F}_{\text {Lorentz }}\right\rangle=0,
\end{aligned}
$$

where angular brackets describe the time-averaging procedure. For standing wave with the extra phase shift $\pi / 2$ between magnetic and electric vectors, Eq. (2) transforms to

$$
\begin{aligned}
& \vec{F}_{\text {Lorentz }}=q[\vec{V}(\vec{r}) \times \vec{B}(\vec{r})] \cos \omega t \cos \omega t= \\
& =\omega \alpha[\vec{E}(\vec{r}) \times \vec{B}(\vec{r})](\cos \omega t)^{2} \\
& \left\langle\vec{F}_{\text {Lorentz }}\right\rangle=\omega \alpha / 2[\vec{E}(\vec{r}) \times \vec{B}(\vec{r})] .
\end{aligned}
$$

If a laser beam is propagating along the normal to a conducting planar surface and is reflected from it, we obtain a standing electromagnetic wave. With account of Eqs. (2) and (3), the pressure induced by electromagnetic field on the oscillating dipoles near the surface with a high reflectivity differs as compared to the case of a relatively low reflecting surface (e.g., a dielectric substrate). The non-zero Lorentz force in a standing electromagnetic wave may result in a quite complicated trajectory of charged particles [18]. But for our case, the peculiarities of the dipoles trajectories in focal point do not define specifics of the pressure induced by electromagnetic wave. The key issue is that dipoles has an extra speed, which results in an extra dynamic pressure.

For the Gaussian profile beam $E \exp \left(-\left(x^{2}+y^{2}\right) / a^{2}\right)$ propagating along $Z$ axis in the focal point (weak dependence on $Z$ coordinate), distribution of forces described by Eqs. (1) and (3) is as follows:

$$
\begin{aligned}
& F_{E S}(x, y)=-E^{2} a^{-2} \exp \left(-2\left(x^{2}+y^{2}\right) / a^{2}\right)(x+y), \\
& F_{\text {Lorentz }}(x, y)=\omega \alpha / 2[\vec{E}(\vec{r}) \times \vec{B}(\vec{r})] \exp \left(-2\left(x^{2}+y^{2}\right) / a^{2}\right) .
\end{aligned}
$$

Taking derivatives from Eq. (4), we can find locations of maximal force values. Coulomb force maximal values are located near the points $x^{2}+y^{2}=a^{2} / 4$, and maximal values of Lorentz force - at point $x=0, y=0$. The forces arise in the focal point for very short time frames, if we apply femtosecond laser pulses. As a result, different types of waves are formed in the focal point [16,17], which satisfy Bernoulli's principle for liquids and gases, when the system reaches the steady state regime. The forces result in dipoles

flows, in higher dynamic pressure associated with the flows and, as a result, in a lower static pressure according to the Bernulli principle.

From a pure practical point of view, standing and travelling waves of high intensity with significant electrostriction pressure can be obtained by focusing the laser light on an Al-covered glass plate and the polished glass one.

\section{Experimental and discussion}

Specific features of laser light (huge coherence length, narrow spectral lasing line width and small beam divergence) allow applying it in variety of laser microscopy schemes. Focusing the light in a small

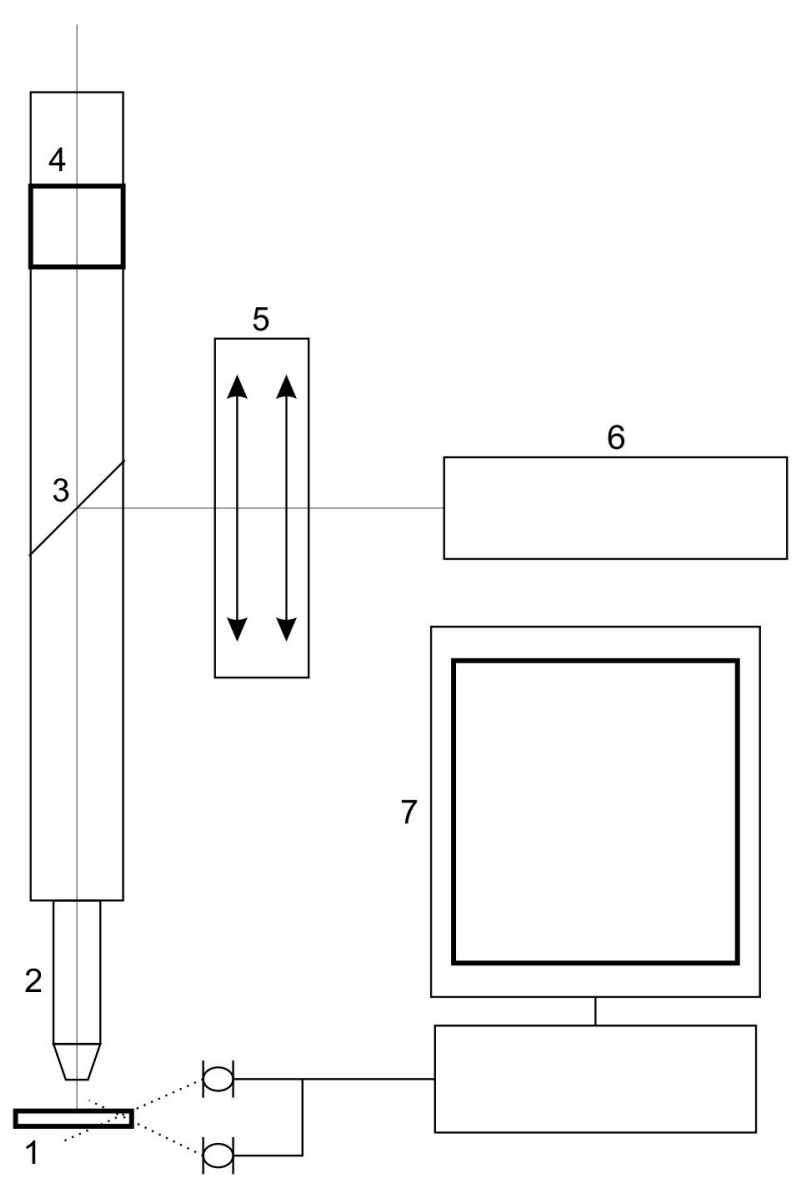

Fig. 1. Optical scheme of laser microscope. 1 - stage with 2 step motors for precise XY positioning, 2 - objective lenses, 3 dichroic mirror for laser beam coupling with microscope. $4-$ notch filter, 5 - beam condensor for matching laser beam waist and objective lenses focal point, 6 - femtosecond laser source with pulse peaker, $7-\mathrm{PC}$ with an interface for stage 1 . 

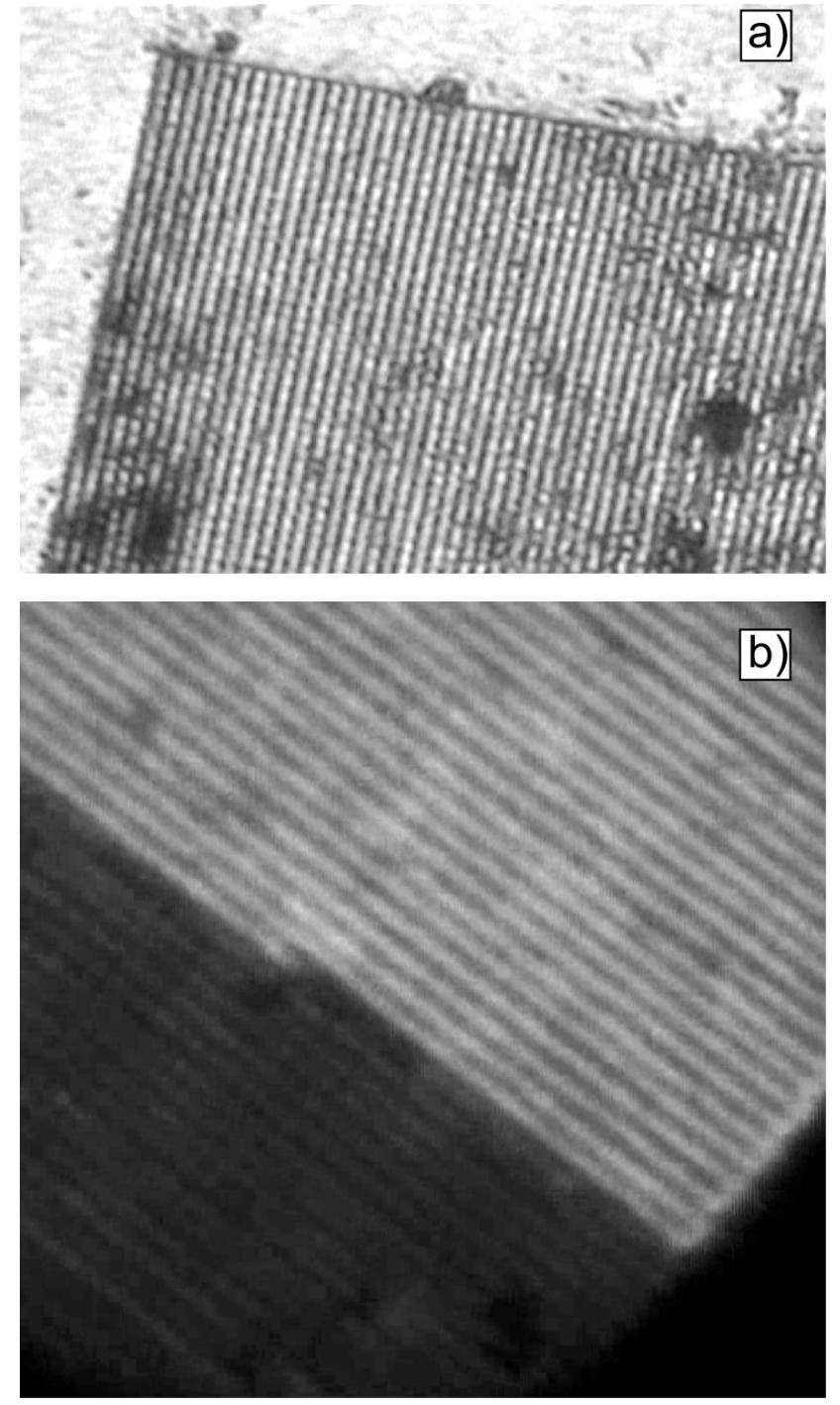

Fig. 2. Microfotographies of two etched rectangular raster structures produced on $\mathrm{Al}$ covered glass plate. The upper picture (a) is taken in the reflected light. The distance between fringes is close to $12.5 \mu \mathrm{m}$. The lower picture (b) is taken in the transient light. The distance between fringes $-2.5 \mu \mathrm{m}$. The upper more bright area of the raster was etched with the laser power twice higher as compared to the lower more dark area.

volume of studied sample, one can register two-photon transitions, Raman scattering or other nonlinear phenomena. Nanostructures (nanoparticles) can be fabricated using a laser coupled with a scanning optical microscope because of extremely high electric field confined in the focal point.

The scheme of our set-up is presented in Fig. 1. Femtosecond tunable light pulses were generated as 120fs pulses within the 800 -nm wavelength range by using a mode-locked Ti:sapphire laser (Mira 900F, Coherent, Santa Clara, Calif.) followed by a pulse peaker to decrease lasing pulses repetition rates down to $100 \mathrm{kHz}$ (Fig. 1). Applying neutral filters with different optical density, we were able to achieve a required level of

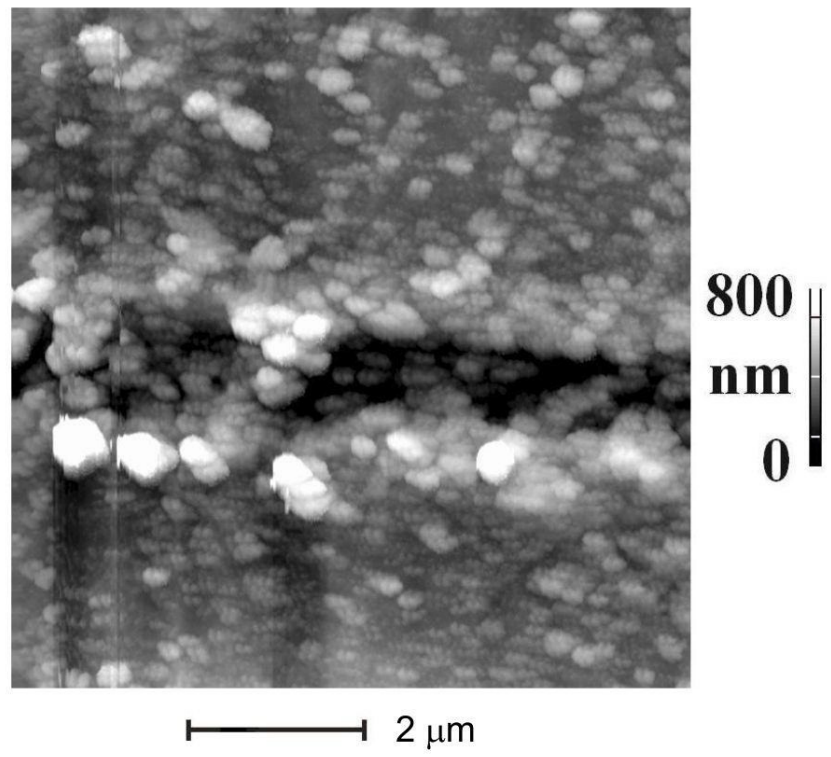

Fig. 3. Atomic force microscopy scan of etched raster structure on a plane glass substrate.

sample surface treatment (e.g., to reach the ablation threshold). The threshold of sample destruction was determined semiquantitatively by removing step-by-step neutral filters from the laser beam. The threshold energy was found to be dependent not only on the objective lens optical strength and vendor but also on everyday laser cavity alignment. The microscope had a coupled to a computer motorized XY stage mounted on the optical table and allowed us to perform different scenarios of nanostructures fabrication on a sample surface. In orderto fabricate the nanoparticles structure on a surface, we performed laser ablation on both polished glass and $\mathrm{Al}$ coated polished glass samples.

Two types of samples were chosen in order to achieve travelling and standing wave cases during laser ablation. A microphotography of the fabricated raster structure of rectangular shape on Al coated glass plate is presented in Fig. 2. The interline distance was equal to $12.5 \mu \mathrm{m}$. Actually, the parallel lines of the raster connect dots, where the surface was exposed to the laser light during $0.1 \mathrm{~s}$. The distance between the dots was equal to $12.5 \mu \mathrm{m}$, too. The space between dots in the raster lines was irradiated using the focused laser beam during table movements when irradiating the sequence of the dots.

Microscope INTEGRA NT-MDT allowed conducting measurements in the atomic force microscopy (AFM) regime and was used to study precise surface topology. AFM spatial resolution reached up to $30 \mathrm{~nm}$. An example of an etched surface of a polished glass studied by AFM is presented in Fig. 3. We can see on the surface both a raster line and a spot where the surface was exposed for the laser light for longer time $(0.1 \mathrm{~s})$. The width of the line is approximately $2 \mu \mathrm{m}$, which is close to the difraction limit of the objective lens in the 


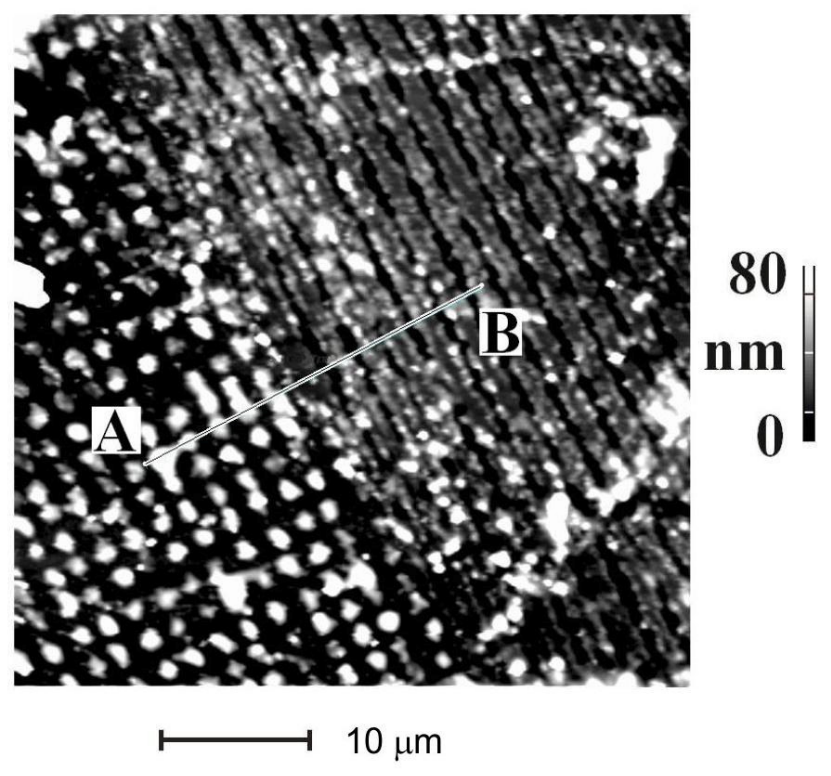

Fig. 4. AFM scan of etched raster structure on $\mathrm{Al}$ covered glass substrate. Microphotography of the structure is presented in Fig. 2 (right picture).

focal point. The surface of the sample is completely covered by submicron size nanoparticles with variety of sizes (the particles with large diameters are closer to the track). The particles are forming craterlike rims alongside the line and dot. The diameter of the rim can be determined using Eq. (4) $\left(x^{2}+y^{2}=a^{2} / 4\right)$. It is clear that the particles are congealed droplets of the melted substance of the sample, which were removed aside the focal point volume during ablation. The surfaces of particles are structured, which seems to indicate some kinetic process adherence of tiny melted droplets into larger ones. The droplets were turned off the melted spot on the substrate surface and their spatial distribution on the surface reflects distribution of the pressure in air, which influence the droplets movement in the time periods exceeding 120 femtosecond laser pulse duration and their final deposition on the substrate suface. The nanoparticles which are congealed droplets of melted substrate are scattered around the etched surface spot at the distances up to $1 \mathrm{~cm}$.

Another AFM scan of an Al-coated glass surface is presented in Fig. 4. The distance between raster lined (and the dots which form the lines) close to $2.5 \mu \mathrm{m}$. We can see that surface has two distinctly different morphology. One part forms a rectangular convex dot grid and another is hollow lines raster. The surface in the area around the point marked in the figure as $\mathbf{A}$ was ablated with an additional neutral optical filter with optical transmittion $50 \%$ in the laser beam as compared to the area of the sample in the vicinity of point $\mathbf{B}$. The microphotography of etched structure in the transient light (Fig. 2, right figure) shows drastic difference in the optical transmittion coefficient between two areas. The
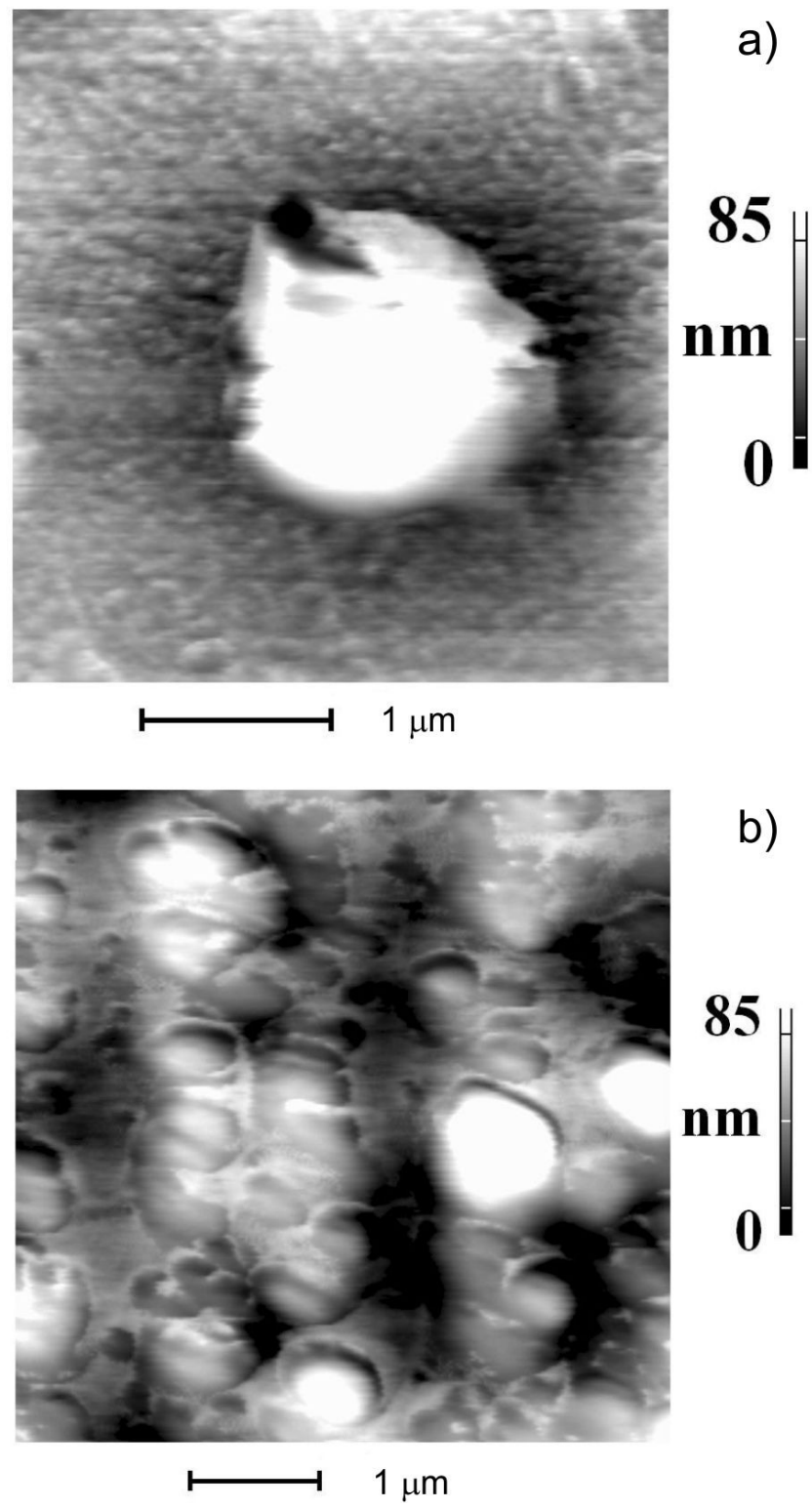

Fig. 5. AFM scan with hihger resolution part of the surface from Fig. 4.

transmittion coefficient of the bright sample area measured using a microphotometer reached $25 \%$, which is a proof of an efficient travelling electromagnetic wave. Of course, we measued the integrated value of optical transmittance averaged in convex and hollow grid lines areas, so the femtosecond light transmittance during the laser ablation was even higher.

AFM scans of Fig. 4 rasters with higher resolution are presented in Fig. 5. As we can see the area aside point B (right figure) is covered by submicron-size nanoparticles similarly to Fig. 3 case. The spot around $\mathbf{A}$ is covered in contrary to $\mathbf{A}$ case by bulk micron-size particles which form $2.5 \mu \mathrm{m}$ periodic rectangular grating. The cross-section of surface profile between the points $\mathbf{A}$ and $\mathbf{B}$ (Fig. 4) is shown in Fig. 6. The point $\mathbf{A}$ corresponds to cross-section zero coordinate and the 


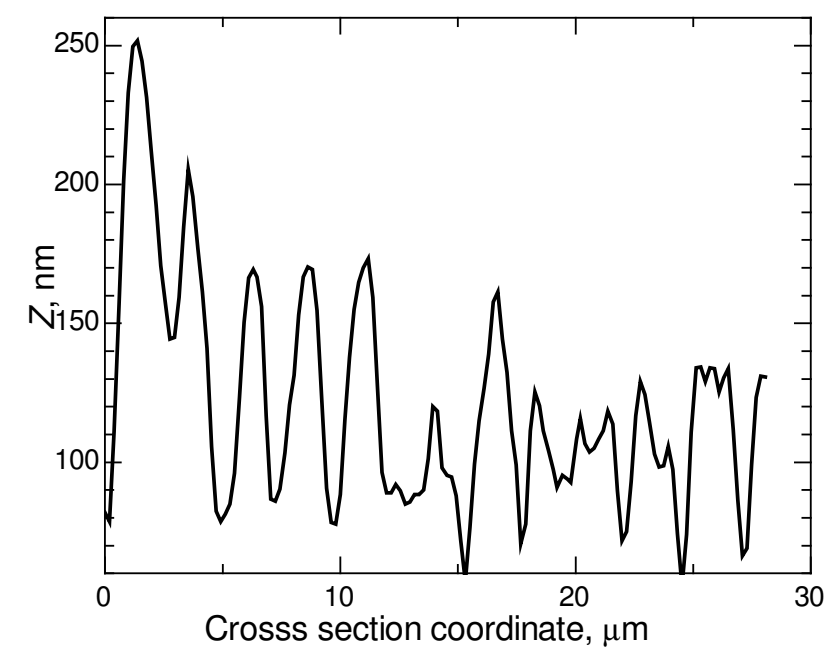

Fig. 6. Height profile for Fig. 4 (the data is taken along the line which connects $\mathbf{A}$ and $\mathbf{B}$ points)
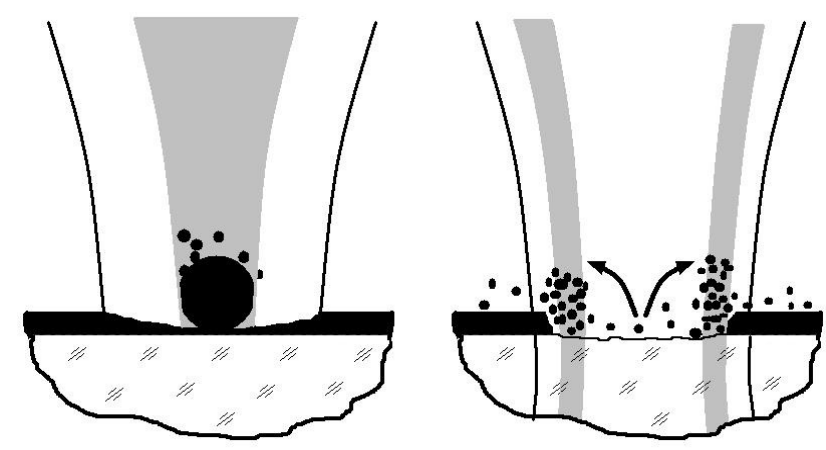

Fig. 7. Electrostriction forces at focused Gauss beam at glass plate covered with an Al layer (marked in black colour). Grey areas depict volumes of maximal electrostriction force.

point $\mathbf{B}-27 \mu \mathrm{m}$. As we can see, the average $Z$ coordinate on the right side of the plot $([13,27]$ cross-section coordinate interval) is appoximately $40 \mathrm{~nm}$ lower as compared to the left side $([0,13]$ cross-section coordinate interval). The substance of the sample surface was melted and removed from the focal point in $\mathbf{B}$ area to long distances. We registered spherical nanoparticles on the substrate scattered to $1 \mathrm{~cm}$ far from the etched area of the sample. We can see variety of spherical submicron-size nanoparticles that cover AFM scanned area (Fig. 5b). By the contrast, the melted substance stuck together forming sole micron-size particles orderly deposited on the surface around the point $\mathbf{A}$. The scenario with a standing light wave was achieved by decreasing slightly the laser power below the Al coating penetration threshold, allowing the coating to reflect radiation. The process is shematically depicted in Fig. 7. The surface of the samples was etched with realization of the standing wave (left side of Fig. 4) and travelling wave (right side of Fig. 4). In the latter case, the $\mathrm{Al}$ coating was melted off the surface in the vicinity of the focal point and transferred aside to the volumes of lower pressure values. This is a reason for nanoparticles to be scattered around volumes of high temperature (electromagnetic wave high density) and low pressure are different, and we have no "optical tweezers" in this case.

\section{Conclusions}

Analyzing the presented results, we can emphasize few key moments. We consider electrostriction as a property of dielectric media that causes them to change their shape under application of a strong electromagnetic field and creates, as a result, an additional pressure. The electrostriction force induced by short laser pulses in isotropic media should be treated with account of Coulomb and Lorentz forces applied to fast oscillating dipoles. The classical ratio for the electrostriction pressure is applicable for relatively steady state regimes with the corresponding laser pulse duration and times of sound waves travelling across the focal point volume.

We have two distinctly different cases of electrostriction pressure for travelling and standing light waves. The latter case of electrostriction has an additional component of the pressure - Lorentz force applied to oscillating dipoles. For Gaussian profile shaped beams, the volumes of maximal electrostriction pressure (for maximum electromagnetic field density) are different, if the wave is travelling, and are the same, if the electromagnetic wave is standing. It is revealed as different surface morphology during femtosecond laser etching planar surfaces. We can obtain either nanopatterning as a result of removing melted substrate substance from the focal point or opposite picture "optical tweezers", when the melted substrate parts are kept together in the focal point.

\section{References}

1. Schaaf P. Laser Processing of Materials (Fundamentals, Applications and Developments). Heidelberg: Springer-Verlag, 2010.

2. Liu B., Hu Z., Che Y., Allenic A., Sun K., Pan X. Growth of $\mathrm{ZnO}$ nanoparticles and nanorods with ultrafast pulsed laser deposition. Appl. Phys. A. 2008. 93. P. 813-818.

3. Vorobyev A.Y., Guo C., Makin V.S., Kokody N.G., Kuzmichev V.M. Extraordinary enhanced absorptivity of gold surface ablated with femtosecond laser pulses. Proc. SPIE. 2008. 7009. P. 700913.

4. Murakami M., Liu B., Hu Z., Liu Z., Uehara Y., and Che Y. Burst-mode femtosecond pulsed laser deposition for control of thin film morphology and material ablation. Appl. Phys. Exp. 2009. 2. P. 042501.

5. Khan S. Z., Yuan Y., Abdolvand A., Schmidt M., Crouse P., Li L., Liu Z., Sharp M., Watkins K.G. Generation and characterization of $\mathrm{NiO}$ nanoparticles by continuous wave fiber laser ablation in liquid. J. Nanoparticle Res. 2009. 11. P. $1421-1427$. 
6. Ullmann M., Friedlander S.K. and Schmidt-Ott A. Nanoparticle formation by laser ablation. $J$. Nanoparticle Res. 2002. 4. P. 499-509.

7. Ko S. H., Choi Y., Hwang D.J., and Grigoropoulos C.P., Chung J., Poulikakos D. Nanosecond laser ablation of gold nanoparticle films. Appl. Phys. Lett. 2006. 89. P. 141126.

8. Hiromatsu K., Hwang D.J., Grigoropoulos C.P. Active glass nanoparticles by ultrafast laser pulses. Micro \& Nano Letters. 2008. 3, No 4. P. 121-124.

9. Eliezer S., Eliaz N., Grossman E., Fisher D., Gouzman I., Henis Z., Pecker S., Horovitz Y., Fraenkel M., Maman S., and Lereah Y. Synthesis of nanoparticles with femtosecond laser pulses. Phys. Rev. B. 2004. 69. P. 144119.

10. Sanz M., Walczak M., de Nalda R., Oujja M., Marco J. F., Rodriguez J., Izquierdo J. G., Banares L., Castillejo M. Femtosecond pulsed laser deposition of nanostructured $\mathrm{TiO}_{2}$ films. Appl. Surf. Sci. 2009. 255. P. 5206-5210.

11. Jeong A.R., Jo W., Ko C., Han M., Kang S.J., Kim M., Park D.Y., Cheong H., Yun H.J. Growth and structural properties of pulsed laser-ablated CuInSe 2 nanoparticles by pulsed-laser ablation and selenization process. Journal of Alloys and Compounds. 2011. 509. P. 8073-8076.

12. Noel S., Hermann J., Itina T. Investigation of nanoparticle generation during laser ablation of metals. Appl. Surf. Sci. 2007. 253. P. 6310-6315.

13. Ashkin A. Acceleration and trapping of particles by radiation pressure. Phys. Rev. Lett. 1970. 24, No 4. P. 156-159.

14. Bonse J., Rosenfeld A., Krueger J. On the role of surface plasmon polaritons in the formation of laser-induced periodic surface structures upon irradiation of silicon by femtosecond laser pulses. $J$. Appl. Phys. 2009. 106. P. 104910.

15. Datsyuk V.V., Pavlyniuk O.R. Maxwell stress on a small dielectric sphere in a dielectric. Phys. Rev. A. 2015. 91, No 2. P. 023826.

16. Rozouvan S. and Dreier T. Polarization-dependent laser-induced grating measurements. Opt. Lett. 1999. 24. P. 1596-1598.

17. Rozouvan S. Polarization dependent electrostrictive grating measurements. J. Chem. Phys. 2007. 127. P. 084501.

18. Bolotovskii B.M., Serov A.V. Details of the motion of charged nonrelativistic particles in a variable field. Physics-Uspekhi. 1994. 37. P. 515-516.

\section{Authors and CV}

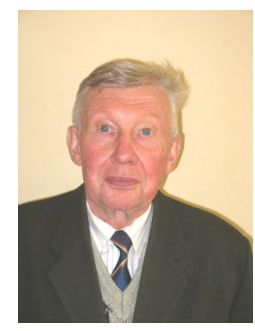

Igor Shaykevich, born in 1934, defended his Doctoral Dissertation in Physics and Mathematics in 1988 and became full professor in 1989. Professor emeritus at Department of Optics of Taras Shevchenko National University of Kyiv. Authored over 300 publications, 15 patents, 2 textbooks. The area of his scientific interests includes spectral ellipsometry of metals and thin films.

\section{Taras Shevchenko National University of Kyiv, Ukraine}

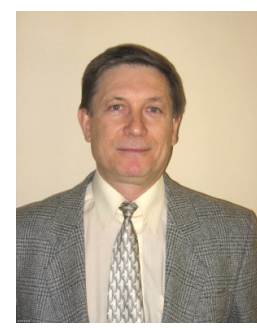

Leonid Poperenko, born in 1950, defended his Doctoral Dissertation in Physics and Mathematics in 1992 and became full professor in 1996. Head of Department of Optics of Taras Shevchenko National University of Kyiv. Authored over 200 publications, 15 patents, 7 textbooks. The area of his scientific interests includes spectral ellipsometry of metals and surface science.

Taras Shevchenko National University of Kyiv, Ukraine

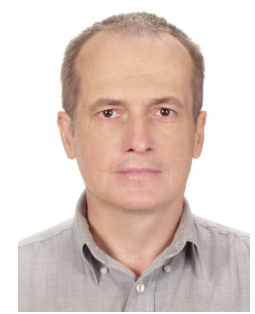

Stanislav Rozouvan, born in 1961, defended his Ph.D. thesis in optics and laser physics in 1995. Scientist at Department of Optics of Taras Shevchenko National University of Kyiv. Authored over 70 publications, 3 patents. The area of his scientific interests includes scanning tunneling microscopy and third-order nonlinear optics.

Taras Shevchenko National University of Kyiv, Ukraine E-mail:sgr@univ.kiev.ua

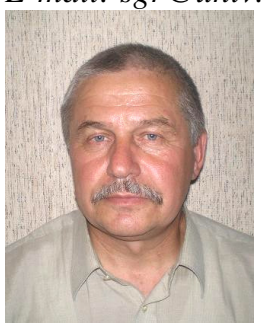

Vasyl V. Prorok, born in 1952, defended his Doctoral Dissertation in Physics and Mathematics in 1982 and became senior researcher in 1986. Senior researcher at Physical Department of Taras Shevchenko University of Kyiv. Authored over 100 publications, 7 patents. The area of his scientific interests includes methods of deposition of thin films, investigation of optical properties of thin films, calculation of optical multilayers, radioecology.

Taras Shevchenko National University of Kyiv, Ukraine 